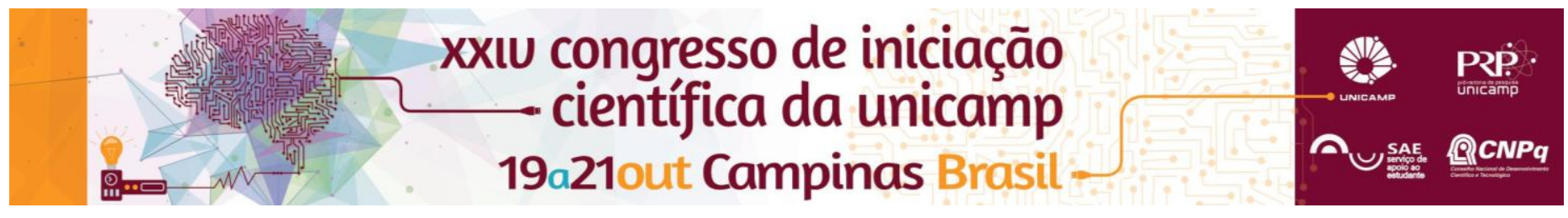

\title{
Burdock root (Arctium lappa, L.) flours produced by air conventional drying and freeze- drying and its application in cookies
}

\author{
Caroline M. Celegatti, Thaísa M. A. Moro, Maria Teresa P. S. Clerici
}

\begin{abstract}
Burdock roots, rich in fructans, has been little used in bakery products. To help motivate increasing its added value, the project aimed to develop burdock root flours by conventional drying (CBF) and freeze-drying (FBF) techniques. We also evaluated its application in cookies with $51 \%$ of whole grain wheat flour and replacing wheat flour (49\%) for CBF in 0 (C0), 5 (C1) and 10\% (C2). Despite the differences in the processes, developed burdock flours had similar composition. $\mathrm{C} 1$ and $\mathrm{C} 2$ cookies had higher fiber content (7.8 and 8.5\%, respectively) than $\mathrm{C} 0(7.0 \%)$. All of them also had stability similar to control in a 30 days shelf life evaluation. Production of cookies until $10 \%$ of CBF generated physicochemical and technological properties in agreement to the control formulation ( $\mathrm{C} 0$ ), and may contribute to expand burdock root uses.
\end{abstract}

Key words: bakery, functional properties, unconventional flours, agribusiness.

\section{Introduction}

Burdock root has chinese origin, is rich in fructans and is considered a functional food but is still little explored and used in bakery products ${ }^{1}$. This study aimed to develop burdock root flours by conventional drying and freeze-drying methods and evaluate conventional burdock flour (CBF) use in cookies produced with whole grain wheat flour.

\section{Results and Discussion}

The physicochemical characteristics ${ }^{2,3}$ of burdock root flours, dried in conventional oven (CBF) and freeze dried (FBF) are presented in Table 1 and Figure 1.

Table 1. Physicochemical properties of burdock root flour by conventional drying (CBF) and freeze-drying (FBF).

\begin{tabular}{|c|c|c|}
\hline Characteristics & $\mathrm{CBF}^{1}$ & $\mathrm{FBF}^{1}$ \\
\hline Moisture (\%) & $10.14 \pm 0.05^{\mathrm{a}}$ & $4.14 \pm 0.23^{b}$ \\
\hline Proteins (\%) & $7.68 \pm 0.51^{n s}$ & $7.91 \pm 0.40^{n .5}$ \\
\hline Ashes $(\%)$ & $3.07 \pm 0.07^{n s}$ & $3.39 \pm 0.04^{n s}$ \\
\hline Lipids (\%) & $0.22 \pm 0.02^{n s}$ & $0.31 \pm 0.02^{\text {ns }}$ \\
\hline Carbohydrates $(\%)^{2}$ & 59.36 & 64.21 \\
\hline Total fibers $(\%)$ & $19.54 \pm 0.34^{\text {ns. }}$ & $20.04 \pm 1.13^{n s}$ \\
\hline Soluble $^{3}$ & $5.64 \pm 0.38$ & 4.24 \\
\hline $\begin{array}{l}\text { Insoluble } \\
\text { Color: }\end{array}$ & $13.90 \pm 0.17$ & 15.80 \\
\hline L & $66.69 \pm 0.57^{b}$ & $89.81 \pm 0.27^{a}$ \\
\hline$a^{*}$ & $5.33 \pm 0.08^{a}$ & $1.23 \pm 0.04^{b}$ \\
\hline $\mathrm{b}^{*}$ & $24.33 \pm 0.27^{\mathrm{a}}$ & $9.54 \pm 0.26^{b}$ \\
\hline
\end{tabular}

'Results expressed as mean \pm standard deviation. Different letters, in the same line, differ by Scott-Knott test $(p<0.05)$; n.s: non-significant. ${ }^{2}$ Digestib
difference between total and insoluble fibers.

Figure 1. Fresh burdock root and burdock root flours by conventional drying (CBF) and freeze-drying (FBF).
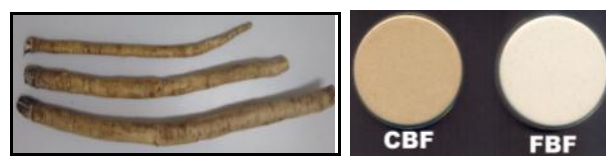

Color differences between flours can be due to processes conditions that generated a darker yellowish tone in CBF. Since apparently there were no variations between flours composition, we chose to use CBF for being a low cost process when compared to freeze-drying. We developed cookies without CBF: control (C0) and with burdock flour, C1 $(5 \%)$ and C2 $(10 \%)$. The results of physicochemical and technological properties ${ }^{2,3}$ are in Table 2.
Table 2. Physicochemical and technological properties of cookies: control (C0) and with burdock flour, C1 $(5 \%)$ and C2 (10\%).

\begin{tabular}{|c|c|c|c|}
\hline Characteristics & $\mathrm{CO}(0 \%)^{1}$ & C1 $(5 \%)^{1}$ & C2 $(10 \%)^{1}$ \\
\hline \multicolumn{4}{|l|}{ Physicochemical } \\
\hline Moisture (\%) & $4.34 \pm 0.19^{\text {n.s. }}$ & $4.76 \pm 0.25^{\text {n.s. }}$ & $4.8 \pm 0.6^{\text {n.s. }}$ \\
\hline Proteins $(\%)$ & $10.29 \pm 0.04^{\text {n.s. }}$ & $10.0 \pm 0.27^{\text {n.s. }}$ & $10.18 \pm 0.12^{\text {ns. }}$ \\
\hline Ashes (\%) & $2.83 \pm 0.02^{n . s .}$ & $2.85 \pm 0.01^{\text {n.s. }}$ & $2.44 \pm 0.68^{\text {n.s. }}$ \\
\hline Lipids (\%) & $20.94 \pm 0.79^{\mathrm{a}}$ & $18.94 \pm 0.24^{b}$ & $19.98 \pm 0.67^{\mathrm{a}}$ \\
\hline Fibers $(\%)^{2}$ & 7.02 & 7.77 & 8.51 \\
\hline Carbohydrates $(\%)^{3}$ & 58.92 & 60.44 & 58.89 \\
\hline \multicolumn{4}{|l|}{ Technological } \\
\hline \multicolumn{4}{|l|}{ Color: } \\
\hline L & $23.38 \pm 0.40^{\mathrm{a}}$ & $22.41 \pm 0.27^{b}$ & $22.19 \pm 0.19^{b}$ \\
\hline$a^{*}$ & $8.03 \pm 0.35^{\mathrm{a}}$ & $6.37 \pm 0.23^{\mathrm{b}}$ & $6.46^{b} \pm 0.13^{b}$ \\
\hline$b^{*}$ & $6.25 \pm 0.32^{\mathrm{a}}$ & $4.81 \pm 0.08^{b}$ & $5.05^{b} \pm 0.08^{b}$ \\
\hline$\Delta \mathrm{E}$ (color difference) & - & 2.40 & 2.31 \\
\hline Texture (N) & $12.69 \pm 5.29^{\mathrm{a}}$ & $24.83 \pm 5.23^{\mathrm{b}}$ & $15.36 \pm 4.01^{\mathrm{a}}$ \\
\hline Especific Volume $\left(\mathrm{cm}^{3} / \mathrm{g}\right)$ & $1.36 \pm 0.17^{\text {n.s. }}$ & $1.25 \pm 0.22^{\text {n.s. }}$ & $1.07 \pm 0.02^{\text {n.s. }}$ \\
\hline Density $\left(\mathrm{g} / \mathrm{cm}^{3}\right)$ & $0.76 \pm 0.09^{\text {n.s. }}$ & $0.83 \pm 0.15^{\text {n.s. }}$ & $0.94 \pm 0.02^{\text {n.s. }}$ \\
\hline Weight (g) & $5.46 \pm 0.14^{\mathrm{a}}$ & $4.88 \pm 0.39^{\mathrm{b}}$ & $5.45 \pm 0.50^{\mathrm{a}}$ \\
\hline TD before and after oven $(\%)^{4}$ & $2.20 \pm 0.40^{\text {n.s. }}$ & $2.11 \pm 0.36^{\text {n.s. }}$ & $2.11 \pm 0.42^{\text {n.s. }}$ \\
\hline TD horizontal and vertical $(\mathrm{mm})^{4}$ & $39.68 \pm 0.66^{\text {n.s. }}$ & $38.98 \pm 0.75^{\text {ns. }}$ & $39.03 \pm 0.71^{n . s}$ \\
\hline Horizontal GD $(\mathrm{mm})^{4}$ & $2.50 \pm 0.79^{\text {n.s. }}$ & $2.02 \pm 0.86^{n . s .}$ & $1.97 \pm 0.85^{n . s}$ \\
\hline
\end{tabular}

Figure 2. Scanned images of cookies: control (C0) and with burdock flour, C1(5\%) and C2(10\%)
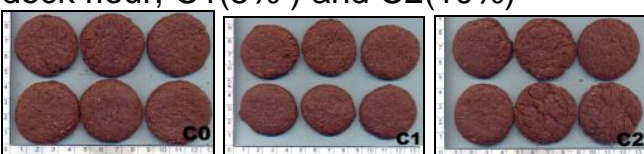

\section{Conclusion}

Although developed with different techniques, flours were only divergent in color. Cookies with until $10 \%$ of CBF produced physicochemical and technological properties similar to the control formulation and may contribute to increase burdock root uses.

\section{Acknowledgement}

The authors thank PIBIC/Unicamp and CAPES for granting the scholarships. We also thank Anaconda Industrial Agrícola de Cereais S.A (São Paulo/Brazil) and Eurogerm (Itupeva/SP) for supplying materials used in this work.

${ }^{1}$ Lin, C.C.; Lu J.M.; Yang J.J; Chuang S.C.; \& Ujiie T. (1996). Antiinflammatory and radical scavenge effects of Arctium lappa. Am J Chin Med., 24(2), 127-37.

${ }^{2}$ AOAC, Association of Official Agricultural Chemists. Official Methods of

Analysis of AOAC international. 18. ed. Gaithersburg: A.O.A.C. International, 2005

${ }^{3}$ AACCI, American Association of Cereal Chemists International. Approved Methods of Analysis. 11. ed. St. Paul: A.A.C.C. International, 2011. 